\title{
A systematic review of family-focused interventions in modifying consumption of sugar and sugar sweetened beverages in children under five years of age
}

\author{
B.C. Foster ${ }^{1}$, N. Shloim ${ }^{2}$, J.B. Moore ${ }^{1}$ and M.A. Zulyniak ${ }^{1}$ \\ ${ }^{1}$ School of Food Science and Nutrition, University of Leeds, Leeds, LS2 9JT and \\ ${ }^{2}$ School of Healthcare, University of Leeds, Leeds, LS2 9JT.
}

Childhood obesity is a global pandemic, with an estimated 41 million children under five years considered overweight or obese ${ }^{(1)}$. In the UK, one in five children are overweight or obese at school entry ${ }^{(2)}$. The consumption of sugar and sugar sweetened beverages (SSB) has been associated with increased weight and adiposity in children ${ }^{(3)}$, which has prompted UK guidance aimed at reducing sugar intake to $5 \%$ total dietary energy ${ }^{(4)}$. This work aimed to systematically review family-focused interventions to reduce children's sugar and SSB consumption.

This review followed PRISMA guidelines and is registered with PROSPERO (CRD42018090940). Principal inclusion criteria were: primary preventative interventions, a family component, and data for sugar and/or SSB intakes in children under 5 years of age. Medline, Embase, PsycInfo, Cinahl and Cochrane databases were searched to $11^{\text {th }}$ January 2018. Risk of bias and study quality were assessed using the American Dietetic Association Quality Criteria Checklist.

Thirty-three studies met the inclusion criteria; 17 were rated positive, 12 neutral, and 4 negative for study quality (Fig. 1). Most studies listed free sugar or SSB intake as secondary outcomes. Free sugar and SSB were both measured in 8 studies. Twenty-five studies measured SSB alone. Overall, 17 studies $(51.5 \%)$, showed a significant effect $(\mathrm{P}<0.05)$ to reduce either free sugar or SSB; 9 studies had significant results for all sugar measurements; while 16 studies reported no effect. Subgroup analysis by age demonstrated that interventions in children before 2 years of age are more likely ( $67 \% ; 9$ studies) to be successful than interventions after 2 years of age ( $46 \% ; 24$ studies).

Overall, positive trends were observed where families were more actively involved and behavioural approaches were adopted, with promising results for interventions within existing child health clinics. These approaches were more effective where existing staff received extra training in behavioural and motivational interviewing techniques.

Children under 2 years responded more favourably to an intervention to reduce sugar intake than children over 2 years, suggesting that an earlier intervention, that discourages introduction of sugar and SSB in children's diets, is a valuable and important area of investigation. Future research should focus on tailoring current interventions for children under 2 years of age.

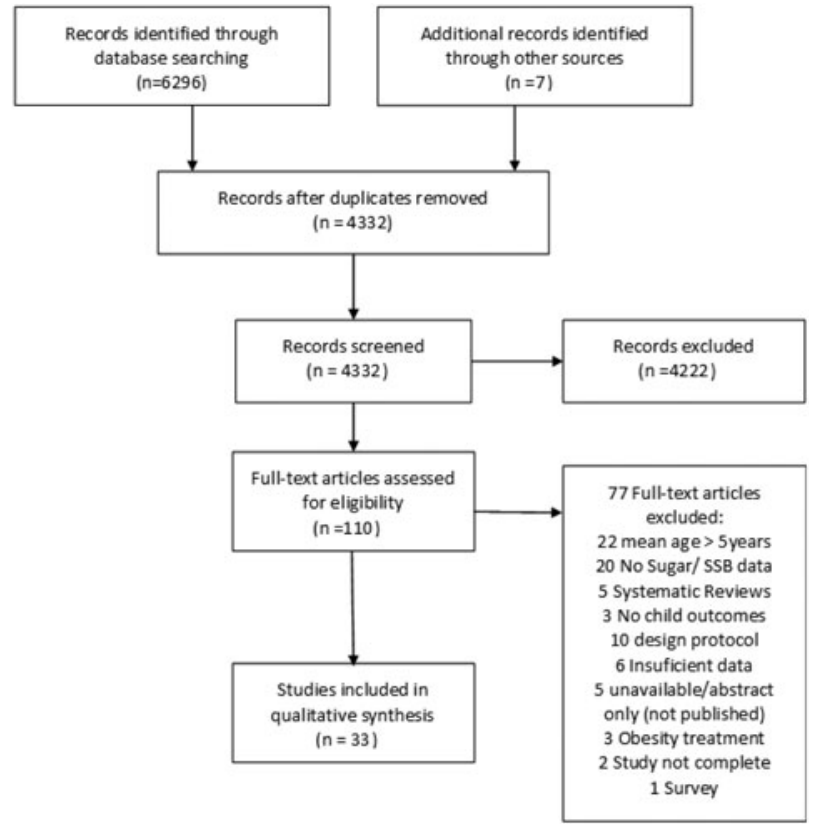

Fig. 1 Flow diagram showing identification and selection process.

1. World Health Organisation (WHO) (2018) Global Strategy on Diet, Physical Activity and Health. http://www.who.int.dietphysicalactivity/childhood/en (accessed April 2018).

2. Public Health England (2015) Childhood Obesity: applying All Our Health. London: Public Health England.

3. Wang J, Shang L, Light K et al. (2015) Appl Physiol Nutr Metab 40, 835-841.

4. Public Health England (2015) Sugar Reduction: The evidence for action. London: Public Health England. 